\title{
Metaanálisis de la seguridad percibida en el transporte público en la era Covid-19
}

\section{Meta-analysis of perceived safety in public transport in the Covid-19 era}

\author{
Cruz García-Lirios ${ }^{\mathrm{a}}$ \\ a*Dra. en Psicología, bundestrans@icloud.com, https://orcid.org/0000-0002-9364-6796, Universidad Autónoma del Estado de México, \\ Benito Juárez, Neza, México
}

Forma de citar: García-Lirios, C. (2021), Metaanálisis de la seguridad percibida en el transporte público en la era Covid-19. Eco Matemático, 12 (1), 107-116

Recibido: 2 de enero de 2021

Aceptado: 15 de febreero de 2021

\section{Palabras clave}

Covid-19, gobierno, gestión, autogestión, cogestión, movilidad, seguridad
Resumen: El objetivo de este estudio fue realizar una revisión sistemática de la literatura para conocer las políticas de movilidad metropolitana y salud periurbana. Se realizó un estudio documental, retrospectivo y meta analítico con una selección no probabilística de fuentes indexadas publicadas del 2019 al 2021 y búsqueda con las palabras clave. Se encontró la prevalencia de riesgos relativos derivados de políticas culturales que sustentan la no exposición a eventos sin consecuencias en la salud de los usuarios. Las comparaciones políticas revelan la prevención de los efectos de los eventos de riesgo en la salud de los usuarios. Las políticas preventivas pueden utilizar el metaanálisis para diseñar programas y protocolos de seguridad.

\section{Keywords}

Covid-19, Self-Management, Co-Management, Mobility, Security

\begin{abstract}
The objective of this study was to carry out a systematic review of the literature to understand the policies of metropolitan mobility and peri-urban health. A documentary, retrospective and meta-analytical study was carried out with a nonprobabilistic selection of indexed sources published from 2019 to 2021 and a search with the keywords. Prevalence of relative risks derived from cultural policies that support non-exposure to events without consequences on the health of users. Political comparisons reveal the prevention of the effects of risk events on the health of users. Preventive policies can use meta-analysis to design safety programs and protocols.
\end{abstract}

*Autor para correspondencia: cgarciali@uaemex.mx 


\section{Introducción}

Hasta mayo de 2021, la pandemia ha matado a seis millones en el mundo y 700 mil en México si se suman los casos de muertes por neumonía atípica y el exceso de muertes desde noviembre de 2019 (OMS, 2021). En este escenario de riesgos de infecciones, enfermedades y muertes debido a Covid-19, las políticas de confinamiento y el distanciamiento social han tenido efectos de rebote al inhibir el desplazamiento y la movilidad de los usuarios del transporte público, pero que agrava la baja calidad del servicio público, que antes de la pandemia era insuficiente (OPS, 2021).

Las políticas de mitigación centran su atención en el confinamiento y distanciamiento social, impactando a la movilidad de personas y a los sistemas de transporte en materia de bioseguridad, calidad y tarifas (Aldana et al., 2201: p. 20). De este modo el estudio de la movilidad anticipa escenarios de exclusión de personas, considerando las políticas de traslado y sus efectos en la bioseguridad de los usuarios (Garcia et al., 2018).

El Área Metropolitana del Valle de México se concentra en la mayor parte del transporte público, pero solo en la Ciudad de México en un $80 \%$ para el $45 \%$ de los usuarios y el $55 \%$ son movilizados por transporte motorizado. En contraste, el Estado de México, ubicado alrededor de la Ciudad de México, concentra el $20 \%$ del transporte público para el 55\% de sus habitantes en la Ciudad de México. Este es un desequilibrio que se traduce en el estado de México y la Ciudad de México (OCDE, 2021). De esta forma, la Ciudad de México recibe alrededor de un millón 735 mil trabajadores y estudiantes del Estado de México (un millón 676 mil), Hidalgo (28 mil), Morelos (14 mil) y Puebla (15 mil). Sin embargo, las quejas por inseguridad han disminuido. En 2012 se presentaron 2441 denuncias, pero en 2018 solo se tramitaron 1564. En la Zona Metropolitana del Valle México (ZMVM) alrededor del 45\% del transporte de la flota de vehículos es el transporte público, seguido del automóvil privado con un $29 \%$ y el uso de taxi con un 11\%. La inflación (4\% acumulado durante 2013) de la gasolina se ha incrementado en un $40 \%$, la prima en un $30 \%$, el diésel en un $48 \%$, mientras que el salario solo se incrementó en un 28\% (INEGI, 2020). La Ciudad de México ocupa los últimos lugares en cuanto a movimiento peatonal con una cifra en torno al 2\%, seguida de la Ciudad de Nueva York con un 10\% y la Ciudad de Londres con un 20\% mientras que en la ciudad de Bombay el $55 \%$ de los despliegues son a pie. En cuanto a la economía familiar, los gastos de transporte se dividen en $50 \%$ para buses o minibuses, 30\% para buses urbanos, $16 \%$ para radio taxis y $2 \%$ para metro. En este contexto, es posible notar que la sostenibilidad del transporte público implica el establecimiento de un sistema de recolección acorde a las capacidades de movilidad periurbana. En 2012 se produjeron alrededor de 580 robos que para 2015 habían aumentado a 600 mil asaltos en transporte público, privado y concesionario. En los casos por demarcación, el 22\% de los casos se registraron en la delegación de Coyoacán, seguido de Gustavo A. Madero con el 15\% de los casos, Iztapalapa con el $14 \%$ y Venustiano Carranza con el $12 \%$ de los delitos. Sin embargo, el transporte público de las ciudades de México se considera inseguro. Se estima que alrededor del 20\% de los usuarios ha dejado de transportar en el sistema público por considerarlo de alto peligro en la Ciudad de México. En 2011, el 78\% de los encuestados dijeron que se sentían inseguros en el transporte público y en 2015 aumentaron al $80 \%$ de los entrevistados. En 2012, el microbús con 1394, seguido del taxi con 641 y el metro con 404 delitos fueron los escenarios de mayor inseguridad. En 2015, se produjeron 983 casos en el minibús, 340 en el metro y 241 en los taxis.

Las políticas de movilidad, particularmente las relativas al trasporte público o concesionado giran en torno a premias socioculturales: aculturación, policultural, multiculturalismo, interculturalismo y transcultural (véase Tabla 1). Se trata de propuestas sobre las cuales se sustentan las decisiones en la materia (Garcia et al., 2015). Es el caso del transporte 
que va de la periferia a la centralidad, atravesando diferentes demarcaciones con sistemas de gestión acordes al tipo de relación entre los flujos migratorios y las comunidades oriundas (Martínez et al., 2019: p. 21).

Tabla 1. Premisas socioculturales de las políticas de transporte metropolitano

\begin{tabular}{|c|c|c|c|c|c|}
\hline & & Ética del cuidado & & Principio de instrumentación & \\
\hline & Simbolismo & Recreación & Comodidad & Satisfacción & uso intensivo \\
\hline Acultural & & & & & $\sqrt{ }$ \\
\hline Policultural & & $\sqrt{ }$ & $\sqrt{ }$ & $\sqrt{ }$ & \\
\hline Multicultural & & $\sqrt{ }$ & $\sqrt{ }$ & $\sqrt{ }$ & \\
\hline Intercultural & $\sqrt{ }$ & & & & \\
\hline Transcultural & $\sqrt{ }$ & & & & \\
\hline
\end{tabular}

Fuente: Estudio elaborado con datos de ingenio

El objetivo de este trabajo es realizar un metaanálisis de la literatura relacionada con la seguridad o inseguridad como consecuencia de la exposición o no exposición de los usuarios del transporte público y su impacto en su integridad física o emocional registrada en 2019 a. 2021 por una gobernanza de la movilidad en la Ciudad de México.

¿Cuáles son los ejes, trayectorias y relaciones entre las variables determinantes y reflexivas del transporte público durante el encierro y distanciamiento social que se inició de marzo de 2020 a marzo de 2021 reportados en la literatura?

La premisa que guía este estudio advierte que las políticas de distanciamiento y confinamiento social amortizaron la demanda de transporte público en el Área Metropolitana del Valle de México, pero a medida que se acumuló la cuarentena, los usuarios decidieron exponerse a contagios, enfermedades y muertes. del Covid-19 influenciado por la comunicación social del gobierno que minimizó los efectos de la pandemia e incluso obligó a los usuarios a reactivar la economía (Tirachi \& Cats, 2020: p. 10). Tal escenario fue desfavorable para la mitigación y contención de la pandemia en el transporte público que traslada a los usuarios de la periferia del Estado de México a la centralidad de la Ciudad de México, propiciando una diversificación de riesgos y aumentando los casos relacionados con neumonía atípica desde el régimen federal y federal (Hernandez et al., 2014). Los gobiernos locales no promovieron las pruebas de detección durante la primera mitad de la crisis de salud (López et al., 2020: p. 21). En consecuencia, la literatura registró hallazgos relacionados con la calidad del servicio vinculado a la transmisión comunitaria del coronavirus, así como los factores que podrían contribuir al control de la pandemia en el transporte público, pero las políticas públicas no consideraron, apostando por la inmunización del rebaño sin siquiera asumir la obligación de usar máscaras y medir el ozono (Zhen et al., 2020: p. 478). De esta manera, la literatura se ha configurado en torno a la aculturación, policulturalismo, multiculturalismo, interculturalidad y transculturales categorías para explicar la resistencia o la cooperación entre los usuarios en el autocuidado y prevención de riesgos (Llamas et al., 2018). 


\section{Materiales y Métodos}

Guerrero y Zapata (2017) advierten que el análisis de datos supone un proceso complejo en el que prevalece una dimensión transversal al considerar la observación de los rasgos de un fenómeno. En este sentido, Se realizó un estudio transversal. Además se documentó una selección de fuentes indexadas a repositorios como Dialnet, Copernicus, Ebsco, Frontiers, Latindex, Publindex, Redalyc , Scielo, Scopus, WoS ,Zenodo \& Zotero de 2019 a 2021, así como las palabras clave: " gobernanza", "gestión", "autogestión", "calidad", "seguridad", "rectoría", "movilidad" y "transporte" (ver Tabla 2).

\begin{tabular}{|c|c|c|c|c|c|c|c|c|c|c|}
\hline & Dialnet & Copérnico & Ebsco & Fronteras & Latindex & Publindex & Scielo & Scopus & Zenodo & Zotero \\
\hline Gobernanza & 35 & 37 & 38 & 33 & 26 & 21 & 15 & 11 & 14 & 17 \\
\hline administración & 30 & 30 & 33 & 28 & 20 & 17 & 11 & 10 & 11 & 13 \\
\hline Movilidad & 27 & 28 & 29 & 24 & 15 & 14 & 8 & 7 & 10 & 11 \\
\hline Calidad & 24 & 26 & 23 & 21 & 13 & 10 & 4 & 5 & 9 & 10 \\
\hline Seguridad & 21 & 20 & 19 & 16 & 10 & 8 & 2 & 3 & 6 & 7 \\
\hline Autogestión & 18 & 17 & 15 & 12 & 9 & 5 & 1 & 2 & 3 & 5 \\
\hline Administración & 14 & 13 & 12 & 10 & 5 & 3 & 1 & 1 & 2 & 3 \\
\hline Transporte & 10 & 11 & 9 & 7 & 3 & 1 & 0 & 0 & 1 & 2 \\
\hline
\end{tabular}

Fuente: Elaborado con estudio de datos

La información fue codificada (véase Tabla 3), siguiendo la metodología de evaluación de la calidad percibida propuesta por Pérez et al., (2017), considerando:

Literatura tipo A para casos de exposición de usuarios a eventos de riesgo (inundaciones, deslizamientos de tierra, incendios, coaliciones, terremotos) y exacerbación de estos por corrupción (negligencia, opacidad, nepotismo, extorsión) con efectos en la salud (estrés, desamparo, hipermetropía, desconfianza).

Literatura tipo B para casos de no exposición a eventos de riesgo y corrupción, pero con efectos en la salud.
Literatura tipo $\mathrm{C}$ para casos de exposición a eventos de riesgo y corrupción, pero sin efectos en la salud.

Literatura tipo D para casos de no exposición a eventos de riesgo y corrupción, pero sin efectos en la salud. 
Tabla 3. Movilidad y seguridad del transporte público en la era Covid-19

\begin{tabular}{|c|c|c|c|c|}
\hline Año & Autor & Políticas & Literatura & Modelamiento \\
\hline 2019 & Martínez et al., & Acultural & $\mathrm{D}$ & Bienestar social $\leftarrow$ Movilidad pública \\
\hline 2019 & Quriz et al., & Transcultural & A & Salud publica $\leftarrow$ Transporte concesionado \\
\hline 2019 & Rosas et al., & Multicultural & $\mathrm{C}$ & Centralidad $\leftarrow$ Movilidad pública \\
\hline 2020 & Lopez et al., & Policultural & B & Bioseguridad en transporte $\rightarrow$ Tiempo de viaje, distanciamiento, ventilación \\
\hline 2020 & Tirachi y Cats & Policultural & B & Movilidad publica $\rightarrow$ Uso de mascarilla \\
\hline 2020 & Zhend & Policultural & B & $\begin{array}{c}\text { Movilidad pública } \rightarrow \text { Tiempo de viaje, hacinamiento, el uso de mascarilla y } \\
\text { ventilación }\end{array}$ \\
\hline 2020 & Quiroz et al., & Multicultural & A & Salud comunitaria $\leftarrow$ Movilidad pública \\
\hline 2021 & $\begin{array}{l}\text { Garibaldi et } \\
\text { al., }\end{array}$ & Transculturall & $\mathrm{D}$ & Gobernanza $\leftarrow$ Bioseguridad en transporte \\
\hline 2021 & $\begin{array}{l}\text { Sandoval et } \\
\text { al., }\end{array}$ & Intercultural & $\mathrm{C}$ & Salud pública $\leftarrow$ Movilidad pública \\
\hline
\end{tabular}

Fuente: Elaborado con estudio de datos. A = Sin exposición y con secuelas, B = Con exposición y con secuelas, C = Sin exposición y sin secuelas, D = Con exposición y sin secuelas

La información se procesó en el paquete estadístico de ciencias sociales (SPSS versión 23.0). Las proporciones de probabilidad (OR) se estimaron con un nivel de significancia del $95 \%$.

El parámetro OR (odds ratio) se utiliza en ciencias de la salud para comunicar los resultados de una investigación, refiriéndose a un coeficiente entre dos probabilidades de ocurrencia de un evento para anticipar un riesgo relativo (RR), aunque en diseños retrospectivos el parámetro OR es más utilizado.

Las estimaciones de RR suponen sesgos de confusión ya que avalan el efecto de diferentes riesgos alusivos a los eventos observados, siendo la estimación de OR ajustada más factible ya que la ponderación de RR ajustada no se puede realizar con la técnica más utilizada como regresión logística, solo binomial. Los modelos logarítmicos reducen el sesgo de confusión al proporcionar un ajuste de RR y OR.
De esta forma, la fórmula para explicar eventos prospectivos y retrospectivos es:

Prospectivo: $\mathrm{OR}=(\mathrm{a} / \mathrm{b})(\mathrm{c} / \mathrm{d})$; estar $\mathrm{a}=$ expuesto, $\mathrm{b}=$ intervenido, $\mathrm{c}=$ no expuesto $\mathrm{y} \mathrm{d}=$ no intervenido

Casos y controles retrospectivos o no apareados: $\mathrm{OR}=(\mathrm{a} / \mathrm{c})(\mathrm{b} / \mathrm{d})$ donde a y c son la exposición de los sujetos a un evento de interés, así como c y d o exposición de los sujetos sin el evento de interés. En ambos estudios, así como en los estudios transversales, el intervalo de confianza que sugiere el grado de variabilidad del OR es fundamental.

Luego, la información fue procesada en una matriz de análisis de contenido con el propósito de extraer los principales conceptos de la agenda de seguridad en el transporte público y establecer hipótesis sobre las trayectorias de las relaciones entre los conceptos seleccionados. Finalmente, se discutieron los alcances y límites del modelo 
propuesto en el marco del cogobierno entre autoridades y usuarios.

La información fue codificada a partir de la técnica Delphi, lo que sugiere la calificación de expertos considerando: 0 para literatura que reporta sin exposición y sin participación del usuario; 1 por la literatura que reporta la exposición, pero no afecta; 2 para literatura que advierte sobre exposición y afectación; 3 para el informe de exposición y afectación.

Una vez realizada la primera ronda de clasificación, los resultados fueron devueltos a los jueces que ajustaron sus criterios de clasificación o mantuvieron su posición. En una tercera ronda final, se eliminaron las puntuaciones más bajas y altas de los jueces que mantuvieron su posición o la modificaron, pero se desviaron del consenso.

\section{Resultados}

La Tabla 4 se refiere a las proporciones de probabilidad de ocurrencia de un evento de riesgo asociado con la corrupción de la administración pública del sistema de transporte público y los efectos sobre la salud ambiental pública de los usuarios.

Tabla 4. Riesgos en torno a la corrupción de la administración pública sobre la salud pública de los usuarios del transporte público (2019 a 2021)

\begin{tabular}{ccccc}
\hline TP & A & B & C & D \\
\hline A & PA & $0,33(0,280,47)$ & $0,66(0,410,83)$ & $0,58(0,380,41)$ \\
\hline B & PM & $0,10(0,070,49)$ & $0,53(0,410,75)$ \\
\hline C & & PP & $0,75(0,400,92)$ \\
\hline D & & & PT
\end{tabular}

Fuente: Elaborado con estudio de datos. $\mathrm{PA}=$ Políticas Aculturales, $\mathrm{PP}=$ Políticas Policulturales, $\mathrm{PM}=$ Políticas Multiculturales, $\mathrm{PI}=$ Políticas Interculturales, $\mathrm{PT}=$ Políticas Transculturales: $\mathrm{A}=$ Sin exposición y con secuelas, $\mathrm{B}=$ Con exposición y con secuelas, $\mathrm{C}=$ Sin exposición y sin secuelas, $\mathrm{D}=$ Con exposición y sin secuelas

La consulta de literatura centra su interés en los efectos de las políticas públicas cuyas dimensiones ubica entre acultural, multicultural, multicultural, intercultural y transcultural.

Las publicaciones revisadas sugieren que las políticas aculturales, multiculturales $\mathrm{y}$ multiculturales están vinculadas a los casos de exposición y no exposición sin consecuencias para la salud, ya que destacan la rectoría del Estado y la administración eficiente del sistema, legitimando incrementos al servicio público sin correspondiente a la calidad y seguridad de estos.

La política cultural se distingue de la multicultural y multicultural en privilegio a las comunidades nativas que habitan las ciudades con respecto a los flujos migratorios que van de la periferia urbana a la centralidad urbana. Es el caso de la disponibilidad, la calidad y el subsidio al transporte, que en la centralidad es dos o tres veces mayor que el costo, la disponibilidad y la calidad del transporte en la periferia.

La política multicultural reconoce la llegada de flujos migratorios por motivos laborales $\mathrm{o}$ educativos, pero no se suscribe a estos sectores y solo garantiza su seguridad con el sistema de videovigilancia que impera en la centralidad urbana. Es decir, da respuesta a las denuncias, pero no les da seguimiento ni busca una solución integral al problema, justificando su inacción por falta de denuncias o avalando su responsabilidad ante las personas, promoviendo la austeridad para no llamar la atención de los ciudadanos.

Las políticas multiculturales sobre transporte público destacan la convivencia y el orden como premisas centrales entre los flujos migratorios y las comunidades nativas, pero ajustando los usos y costumbres de los visitantes a las leyes de la centralidad urbana. De esta manera, un delito cometido en una unidad que va de la periferia a la centralidad se considera una responsabilidad de la autoridad federal más que local. O la demarcación 
de responsabilidades ante la desregulación del transporte en la periferia urbana.

En cambio, las políticas públicas de movilidad desde enfoques interculturales y transculturales resaltan la exposición y no exposición de los usuarios a eventos de riesgo derivados del cambio climático y agravados por la corrupción local con secuelas a corto, mediano y largo plazo en la salud mental y física de los usuarios, reflejándose en la desconfianza hacia sus autoridades y los conflictos con las dependencias del gobierno a cargo de la gestión y administración del sistema.

La política intercultural busca una administración corresponsable entre las entidades gubernamentales de centralidad y periferia, previniendo e impartiendo justicia a los delitos cometidos en el sistema de transporte público y asistencia a las víctimas de riesgo, corrupción y delincuencia, pero considerando una relación de diálogo permanente entre gobierno y educación. y sectores laborales, sindicatos y organizaciones civiles para el mejoramiento de la calidad y seguridad del sistema.
Con base en el aumento de eventos de riesgo y corrupción, la política transcultural busca reducir los riesgos en el transporte público a partir de la eliminación de los límites administrativos para la descentralización y la inversión; generar oportunidades de salud, educación y empleo en la periferia; capacitar a empleados y policías, así como promover vidas libres de violencia y riesgos para los usuarios.

Con base en estas distinciones, es posible notar que la literatura parece enfocarse en los efectos de las políticas multiculturales y transculturales que reconocen la exposición de los usuarios al riesgo y la corrupción, pero no conocen ni reconocen secuelas mínimas en su salud física y mental, dirigiendo sus discursos hacia el subdistrito para atender al bienestar del usuario.

Las asociaciones entre los tipos de políticas y los tipos de literatura permitieron realizar modelos para investigar su composición estructural (ver Tabla 5).

\begin{tabular}{|c|c|c|c|c|c|c|c|c|c|c|}
\hline & PA & PP & $\mathrm{PM}$ & PI & $\mathrm{PT}$ & & A & B & $\mathrm{C}$ & $\mathrm{D}$ \\
\hline $\mathrm{PA}$ & 1.000 & 0,329 & 0,362 & 0,310 & 0,304 & A & 1.000 & 0,320 & 0,374 & 0,320 \\
\hline $\mathrm{PP}$ & & 1.000 & 0,370 & 0,341 & 0,310 & B & & 1.000 & 0,381 & 0,354 \\
\hline PM & & & 1.000 & 0,305 & 0,302 & $\mathrm{C}$ & & & 1.000 & 0,315 \\
\hline PI & & & & 1.000 & 0,313 & $\mathrm{D}$ & & & & 1.000 \\
\hline PT & & & & & 1.000 & & & & & \\
\hline
\end{tabular}

Fuente: Elaborado con estudio de datos. PA = Políticas Aculturales, $\mathrm{PP}=$ Políticas Policulturales, $\mathrm{PM}=$ Políticas Multiculturales, $\mathrm{PI}=$ Políticas Interculturales, $\mathrm{PT}=\mathrm{Políticas}$ Transculturales: $\mathrm{A}=$ Sin exposición y con secuelas, $\mathrm{B}=$ Con exposición y con secuelas, $\mathrm{C}=$ Sin exposición y sin secuelas, $\mathrm{D}=\mathrm{Con}$ exposición y sin secuelas 
Una vez establecidas las probabilidades de riesgo relativo en torno a los eventos de riesgo y sus efectos en la salud local de los usuarios del transporte público, se procedió a estimar su estructura de trayectorias de relación, considerando las posibles combinaciones entre los tipos de políticas y los tipos de literatura.

Es posible apreciar que las trayectorias de relaciones reflexivas entre los tipos de políticas y los tipos de literatura sugieren un riesgo relativo moderado. Es decir, la literatura parece registrar diferentes exposiciones o no a eventos de riesgo con diferentes efectos o no a la salud de los usuarios, destacando las alusivas a la no exposición y no impacto que en las políticas culturales se establecen como agenda metropolitana.

Con el propósito de observar el surgimiento de una política común a las políticas aculturales, poli, multi, inter y transcultural, se realizó la estimación de una estructura confirmatoria.

Es posible observar el surgimiento de una política de salud configurada desde las perspectivas acultural, poli, multi, inter y transcultural, sugiriendo una relación de dependencia entre estos elementos que explicaría la composición de la política de salud en términos de atención a los efectos del riesgo. eventos en el transporte público y su efecto en la salud de los usuarios durante el período 2019 al 2021.

Las relaciones entre los tipos de políticas y los tipos de literatura permitieron realizar un análisis de las relaciones de dependencia entre los elementos.

La estructura de las relaciones de dependencia entre los tipos de políticas y los tipos de literatura muestra que las políticas culturales, señaladas por la literatura que reporta la exposición o no de los usuarios del transporte público en eventos de riesgo y que tuvieron efectos sobre su salud o no, influyeron en la población local. políticas de salud, indicadas por los cuatro tipos de exposición y secuelas enunciadas.

Para observar la estructura de la razón de probabilidad, se establecieron los rangos y la probabilidad de ocurrencia.

Es posible ver que existe una mayor proporción de probabilidad de que los principios de la política cultural afecten a la política de salud local. A continuación, los fundamentos de las políticas policulturales y multiculturales estarían influyendo más que presupuestos interculturales y transculturales, sugiriendo que el transporte público es un instrumento de gestión y gestión de riesgos relacionados con los efectos de los eventos de salud.

\section{Conclusiones}

El objetivo de este trabajo fue realizar un metaanálisis del transporte público en relación con las medidas de prevención de los riesgos de contagio, enfermedad y muerte por Covid-19. Se encontró la prevalencia de políticas públicas de orden acultural, policultural, multicultural y transcultural donde se reportaron efectos aleatorios homogéneos de factores relacionados con la calidad del servicio de transporte al anticipar una crisis de salud. En relación con el estado del arte, se recomienda realizar una evaluación de las políticas de transporte público durante el tiempo que dure la pandemia para poder definir responsabilidades administrativas respecto a la mitigación del Covid-19. Dichos hallazgos permitirán llevar a juicio a quienes, contando con la evidencia científica, tomaron decisiones encaminadas a la inmunización del rebaño, exponiendo a los usuarios del transporte público a riesgos de infección, enfermedad y muerte.

El aporte del presente trabajo al estado de la cuestión radica en un metaanálisis de las contribuciones a la incidencia de eventos de riesgo asociados a la corrupción en la salud pública ambiental, aunque el diseño de la investigación limita los hallazgos al escenario local, sugiriendo la 
extensión del trabajo a otras metrópolis de América Latina. El metaanálisis de estudios de transporte público comparó las políticas metropolitanas ante eventos de riesgo y sus efectos en los usuarios. La revisión destaca el surgimiento de sistemas aculturales, poli, multi, inter y transculturales en la medida en que el transporte público fue diseñado como un instrumento de gestión del desarrollo rural en referencia a la ciudad.

Sin embargo, los eventos de riesgo han resaltado sus efectos sobre la salud pública ambiental, el surgimiento de una ética de cuidado ambiental y han destacado una respuesta colectiva de los usuarios conocida como posmaterialismo para explicar el uso intensivo del transporte ya no solo por su imagen y destino, sino por la idea de considerar que el bienestar económico genera más necesidades de traslado y albergue. Las consecuencias del impacto de los eventos de riesgo y las políticas públicas en la salud de los usuarios no se reconocen explícitamente en el enfoque acultural, multicultural y multicultural, pero es en el enfoque intercultural y transcultural donde prevalece la simbolización de estas secuelas en las enfermedades, accidentes o contingencias.

Las líneas de investigación relacionadas con las políticas de transporte público metropolitano y su relación con los eventos de riesgo nos permitirán anticipar escenarios contingentes y diferenciadores entre actores políticos y sociales, así como entre los sectores público y privado. La técnica metaanalítica ha permitido diferenciar la literatura que trata sobre políticas públicas, eventos de riesgo de exposición y probables secuelas en los usuarios, pero un análisis de las trayectorias de relaciones entre estas variables permitirá observar la eficiencia de los programas y la efectividad de estos. las estrategias para reducir riesgos, prevenir accidentes y atender las enfermedades derivadas del traslado de usuarios de la periferia urbana al centro de la ciudad.

López et al. (2020: p. 21), Tirachi \& Cats (2020: p. 10) y Zhen et al., (2020: p. 478) informaron que la literatura consultada advirtió sobre la prevención de riesgos de contagio en transporte público con la implementación de máscaras, ventilación y distanciamiento. Tales medidas corresponden a modelos de políticas de transporte policulturales, multiculturales y transculturales, pero en el presente trabajo se encontró que fueron las políticas aculturales las que tuvieron un mayor efecto aleatorio homogéneo. Esto es así porque la literatura consultada parece advertir que las políticas donde los usuarios se adaptan al sistema de manejo de pandemias sin medidas preventivas, buscando la inmunidad del rebaño, fueron más decisivas que las políticas de solidaridad y cooperación en el uso de dispositivos de protección. Las líneas de estudio relacionadas con la evaluación de las políticas de transporte público con respecto a la mitigación de la pandemia deberán aclarar la responsabilidad de las autoridades en los diferentes niveles con respecto a la prevención de contagios, enfermedades y muertes.

\section{Referencias}

Garcia, C., Carreon, J., Hernandez, J., Rivera, B. L., Aguilar, J. A. \& Rosas, F. J. (2015). Diferencias perceptuales ante el riesgo a las aglomeraciones al transporte público y concesionado. Iberoforum, 10 (19), 112-131 https://ibero. $\mathrm{mx} /$ iberoforum/19/pdf/ESPANOL/5.\%20 GARCIA\%20LIRIOS\%20ET\%20AL\%20 VOCES $\% 20 Y \% 20 C O N T E X T O S \% 20$ NO. $\% 20$ 19.pdf

Garcia, C., Limon, G. A., Sandoval, F. R., Bustos, J. M. y Juarez, M. (2018). Exploratory factors structural of the habitus mobility. Interconectando Saberes, 3 (6), 37-46 https:// is.uv.mx/index.php/IS/article/view/2568/4497

Garibaldi, S. A., Carreon, J. \& Garcia, C. (2021). Modelling of the mobility habitus in the public transport system with low $\mathrm{CO} 2$ emission mechanics in the center of Mexico. Journal Advances in Mechanics, 9 (2), 82-95

Guerrero, E. J. \& Eslava, R. A. (2017). Aplicaciones de software científico para el análisis de datos en diseños mixtos de investigación. Ecomatemàtico, 
8 (1), 106-115 https://revistas.ufps.edu.co/index. php/ecomatematico/article/view/1481/1387

Hernandez, J., Carreon, J., Morales, M. L., Aguilar, J. A. \& Garcia, C. (2014). Complejidad sociopolítica del transporte público. Implicaciones para el desarrollo local sustentable. Razón \& palabra, 86, 1-27 http:// www.razonypalabra.org.mx/N/N86/V86/12 HernandezCarreonMoralesAguilarGarcia_V86. pdf

Instituto Nacional de Estadística, Geografía e Informática (2020). XIV Censo Nacional de Población y Vivienda. México: INEGI https:// www.inegi.org.mx/

Llamas, B., Bustos, J. M. \& García, C. (2018). Especificación de un modelo para el estudio de habitus de movilidad. Investigación Académica Sin Frontera, 11 (27), 1-22 https:// revistainvestigacionacademicasinfrontera. unison.mx/index.php/RDIASF/article/ view/174/170

López, N., Stern, D., Pérez, C., González, R., Canto, F. y Barrientos, T. (2020). Revisión rápida: Contagio por transporte de infecciones respiratorias agudas en el público y medidas para mitigarlo. Salud Publica de México, Diseminación Rápida. https://saludpublica.mx/ index.php/spm/article/view/11601/11880

Martínez, E., Sánchez, A. y García, C. (2019). Gobernanza de la calidad de vida y el bienestar subjetivo. Ajayu, 7 (1), 121-139

Organización Mundial de la Salud(2021). Estadística de coronavirus SARS CoV-2 y enfermedad de Covid-19 en el mundo. Ginevra: WHO https:// www.who.int/es

Organización para el Desarrollo Económico y la Cooperación (2020). Estadística por país. Bruselas: OCDE http://www.oecd.org/

Organización Panamericana de la Salud (2021). Estadística de coronavirus SARS CoV-2 y enfermedad de Covid-19 en América. Nueva York: OPS https://www.paho.org/en

Pérez, M. O., Orlandoni, G., Ramoni, J. \& Valvuena, M. (2017). Evaluación de la calidad en la prestación de servicios de salud por medio de series de tiempo enmarcados en la metodología seis sigma. Ecometemático, 8 (1), 73-77 https:// revistas.ufps.edu.co/index.php/ecomatematico/ article/view/1387/1358

Quiroz, C. Y., Bustos, J. M., Juarez, M., Bolivar, E. \& Garcia, C. (2020). Exploratory factor structural model oof the perception of mobility of bikeway. Propositos y Representaciones, 8 (1), e422 http://dx.doi.org/10.20511/pyr2020. v8n1.422

Quiroz, C. Y., Bustos, J. M., Juarez, M., Bolivar, E., Quintero, M. L. \& Garcia, C. (2019). Red metanalytical retrospective of the policies in the holding of risk of transportation in the Mexico City. Net Journal Social Science, 7 (4), 92-100 http://www.netjournals.org/pdf/ NJSS/2019/4/19-034.pdf

Rosas, F. J., Gomez, D. A. \& Garcia, C. (2019). Especificación de un modelo para el estudio de la percepción de la movilidad. Eureka, 16 (1), 177-188 https://psicoeureka.com.py/sites/ default/files/articulos/eureka-16-1-17.pdf

Sandoval, F. R., Molina, H. D. \& Garcia, C. (2021). Metanalytic network retrospetive of public transport and its effects on the governance of health. International Journal of Social Science, 9 (1), 8-18 https://doi.org/10.5958/24542679.2021 .00002 .5

Tirachi,A. y Cast, O. (2020). Covid-19 y el transporte público: evaluación actual, perspectivas e investigación de necesidades. Journal de transporte público, 22 (1), 1-21 https:// scholarcommons.usf.edu/cgi/viewcontent. cgi article $=2009 \&$ context $=$ jpt

Zhen, J., Chan, C., Schoonees, A., Apatu, E., Thabane, L. y Young, T. (2020). Transmisión de virus respiratorios cuando se usa el transporte público terrestre: una revisión rápida para informar las recomendaciones de salud pública durante la pandemia de Covid-19. The South African Medical Journal, 110 (6), 478-483 http://www.samj.org.za/index.php/samj/article/ view/12943/9322 